\title{
Visualizing Immune Surveillance in Tumor Microenvironment with Two-photon Microscopy
}

\author{
C. Tong, J. Myers, D. Barkauskas, C. Su, H.-L.R. Liou, S. Intzes and A.Y. Huang \\ Division of Pediatric Hematology / Oncology, Department of Pediatrics, Case Western Reserve \\ University School of Medicine, WRB 6528, 2103 Cornell Road, Cleveland, OH 44106-7288 USA
}

Timing and location of sequential immune cell associations is critical in shaping host immune response toward inflammation or tolerance. Such determination can be crucial in the pathogenesis and elimination of cancer. The precise mechanisms by which clinically evident tumors achieve immune evasion remain a subject of intense investigation. Interestingly, tumor-specific immunity often fails to develop despite the presence of tumor-associated antigens and lymphocytes specific for them in tumor-bearing hosts, suggesting an important role for tumor-infiltrating microenvironment. Indeed, accumulating data indicate that some tumor cells can present and activate antigen-specific $\mathrm{T}$ cells resulting in tumor elimination and protection against subsequent tumor challenges [1]. In contrast, DCs within tumor microenvironment are reported to be responsible for tolerance induction [2-5]. These studies serve to highlight the importance of tumor-infiltrating microenvironment in dictating the ultimate outcome of tolerance or immunity against cancer cells. Traditional in vitro manipulations and gross in vivo experimental approaches to study host - tumor interactions are often inadequate to address the hallmark properties of involved immune cells which include homing, migration, and dynamic interaction with sing-cell specificity [6, 7]. Popular whole-body in vivo imaging modalities, such as MRI, CT and bioluminescence imaging, often lacks the single-cell resolution necessary to discern immune cell specificity. On the other hand, high-resolution microscopic examinations of fixed tissue specimen or live cell suspension in vitro are devoid of either the dynamic information or the 3-D architectural context of the tissue.

Recent new insights into in vivo immune response generation have come from studies involving the use of intravital 2-photon laser scanning microscopy (2P-LSM), a technique we and others have utilized over the past several years to visualize immune cell trafficking and interaction in different anatomical locations within anesthetized experimental mice [8-12]. This novel imaging modality allows deep tissue visualization ( $>300 \mathrm{um}$ ) with single-cell resolution $(<1 \mathrm{um})$, thus enables direct observation of immune cell behavior in intact lymphoid and other tissues at a spatial-time resolution suitable for tracking cellular migration and interactions [8, 11-18]. As an example of the utility of this experimental approach, we have previously investigated in vivo mechanisms mediating efficient cellular cooperation among $\mathrm{CD} 8^{+} \mathrm{T}$ cells, $\mathrm{CD} 4^{+} \mathrm{T}$ cells and $\mathrm{DC}$ during primary adaptive immune response in the lymph node. We have uncovered a critical role that inflammatory chemokines CCL3 / CCL4 play in the early recruitment of naïve $\mathrm{CD}^{+} \mathrm{T}$ cells to sites of ongoing $\mathrm{CD}^{+} \mathrm{T}$ cell $-\mathrm{DC}$ interaction [14]. Application of 2P-LSM has helped to uncover this previously under-appreciated role of inflammatory chemokines in the generation of primary adaptive immune response in LNs. Currently, our laboratory is focused on understanding the role of tumor microenvironment in shaping immune repertoire towards tolerance, and developing strategies to modify tumor microenvironment for the generation of effective anti-tumor immunotherapy approaches. An example of early imaging data in this effort is represented in Figure 1, in which we are able to track dynamic data of naïve lymphocytes surrounding the primary tumor nodules and associated tumordraining lymph nodes. These and other data will be presented during the Symposium to highlight the 
importance of tissue architecture and micro-environment in shaping the overall anti-tumor immune response.

References

[1] Horna, P., et al., Blood 107 (2006) 2871.

[2] Sotomayor, E.M., et al., Blood 98 (2001)1070.

[3] Ghiringhelli, F., et al., J Exp Med. 202 (2005) 919.

[4] Cuenca, A., et al., Cancer Res. 63 (2003) 9007.

[5] Alard, P., et al., Int Immunol. 15 (2003) 945.

[6] Luster, et al., Nat Immunol. 6 (2005) 1182.

[7] Lucignani, G., et al., Trends Biotechnol. 24 (2006) 410.

[8] Huang, A.Y., et al. Immunity 21 (2004) 331.

[9] Huang, A.Y., Biol. Blood and Marrow Transpl. 13 (2007) 111.

[10] Miller, M.J., et al., Science 296 (2002) 1869.

[11] Mempel, T.R., et al., Nature 427 (2004) 154.

[12] Witt, C.M., et al., PLoS Biol. 3 (2005) 160.

[13] Germain, R.N., et al., Semin Immunol. 17 (2005) 431.

[14] Castellino, F., Huang A.Y., et al., Nature 440 (2006) 890.

[15] Bajenoff, M., et al., J Exp Med. 203 (2006) 619.

[16] Qi, H., et al., Science 312 (2006) 1672.

[17] Chieppa, M., et al., J Exp Med. 203 (2006) 2841.

[18] Tadokoro, C.E., et al., J Exp Med. 203 (2006) 505.

[19] This research is supported by Investigator Awards from Cancer Research Institute, the Dana Foundation, Gabrielle's Angel Foundation, Hyundai Motors of America, and the St. Baldrick's Foundation. Dr. Huang is the recipient of St. Baldrick's and Hyundai Scholar Awards, and Dr. Intzes is the recipient of St. Baldrick's Fellow Award.
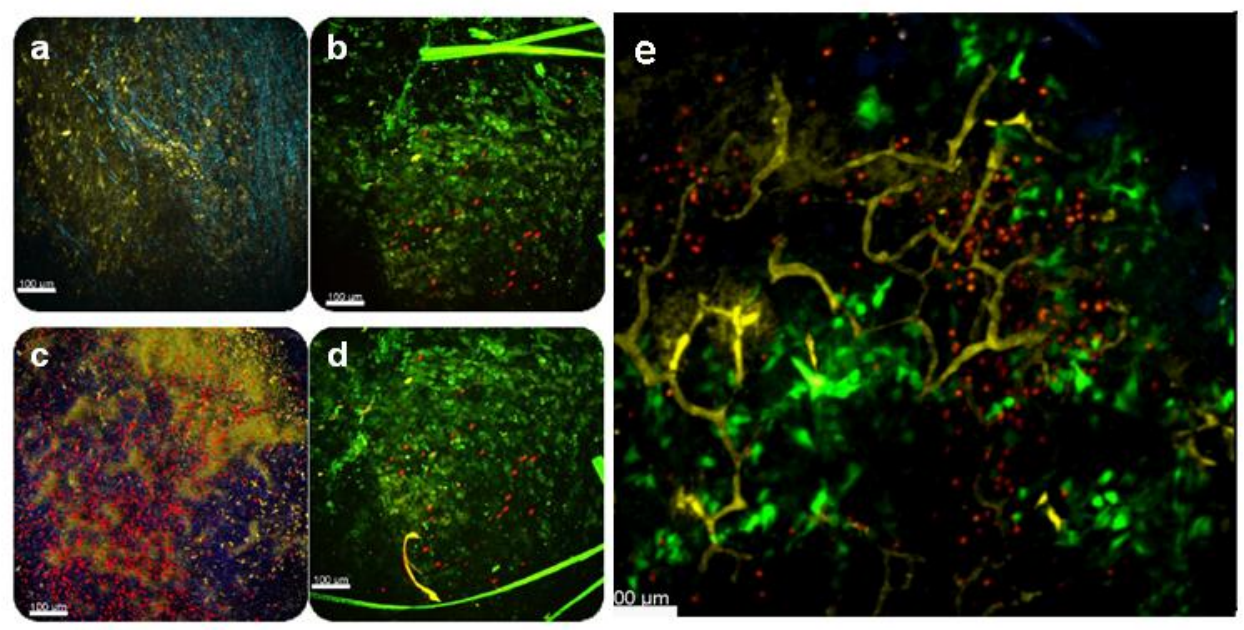

Fig. 1. Two-photon images of T cell infiltrate surrounding CT26-XFP tumor microenvironment. A) Three days after CT26-YFP cells (murine colon tumor model in BALB/c background; yellow) were injected SQ in BALB/c mice; the tumor nodule was imaged using 2-photon microscopy. Blue: collagen fiber ( $2^{\text {nd }}$ harmonic signal) associated with stroma; B, D) $1 \times 10^{7}$ naïve T cells (red, SNARF1) were adoptively transferred into CT26 tumor-bearing mice. 2-photon imaging was performed at the CT26-YFP (green/yellow) tumor sites. C) LN draining tumor injection sites containing numerous injected lymphocytes (red). Note yellow signals arising from metastatic CT26-YFP and phagocytes engulfing tumor debris. E) Similar experiment in draining $\mathrm{LN}$ as in $\mathrm{C}$, with blood vessels highlighted in yellow (TRITC-dextran) and CT26-YFP in green. 